intimate as that of the soul to the body; we cannot get between them even in thought, but the difference is one of kind and not of degree." There is much in the volume about the wonders of the inorganic domain, especially under the eyes of modern chemists and physicists, but the refrain is always what Tyndall called "the mystery and the miracle of vitality." Thus, to mention half of the fascinating studies, we have discussions of "The Breath of Life," "The Living Wave," "The Baffling Problem," "Scientific Vitalism," and "The Vital Order."

It is not easy to describe the life of the bee-hive without the postulate of psychical organisation, what Maeterlinck called the Spirit of the Hive; so to Burroughs it appears necessary to recognise a more than physico-chemical unity of the organism, in which the cells are the bees, and thus he speaks of the Spirit of the Body. But this vitality is potential in all matter, though it finds opportunity to manifest itself with emphasis in protoplasm. Vitality begins in the inmost sanctuary of the molecules, "but whether as the result of their peculiar and very complex compounding or as the cause of the compounding-how are we ever to know?" "The striking essay entitled "A Bird of Passage" develops the idea that life plays a very small part in the total scheme of things, "the great cosmic machine would go on just as well without it." Yet it is only in the highest expressions of life that the total scheme of things acquires any meaning at all. And the author ends with the thought, which he knows to be beyond science, that there is a kind of universal mind pervading not only living matter, but the stuff of which the whole world has been spun. As the reader is warned in the preface, there is considerable reiteration in the course of the essays, but with a writer like Burroughs the impression left is that of music with a recurrent theme.

$$
\text { J. A. T. }
$$

\section{DIOPHANTINE ANALYSIS.}

Mathematical Monographs. No. 16, Diophantine Analysis. By R. D. Carmichael. Pp. vi + I 8 . (New York: J. Wiley and Sons, Inc.; London : Chapman and Hall, Ltd., I9I5.) Price $5 s .6 d$. net.

THE remarkable thing about Diophantine ana1 lysis is that, although it is quite respectably old, it is still in that stage where the amateur is on an equal footing with the professional. If it be true, as we are inclined to think, that Fermat's last theorem admits of a Diophantine proof, this is as likely to be discovered by a schoolboy as by a professor steeped in all the lore of modern analysis.

Prof. Carmichael's book is welcome because it gives, either in the text or in the examples, a great deal of the actual results hitherto obtained; and the author has done something towards sorting out these results and adumbrating a real theory. In NO. $245 \mathrm{I}$, VOL. 98 ] this respect chap ii. (on multiplicative domains) is the most valuable. Chap. v. gives a brief but up-to-date account of what is known about Fermat's last theorem. Important sections are those which treat of Fermat's methods of "descent" and of "double equations"; these, at any rate, are definite processes capable of extension to various cases.

The weak point of the book, in our opinion, is that the author never looks at a problem from a geometrical point of view. Of course, in the last resort, geometry is irrelevant; but in research it is very valuable. For instance, let $\mathrm{F}(x, y, z)$ be a homogeneous cubic; then from the theory of curves we can conclude that if $\mathrm{F}=\mathrm{o}$ has an integral solution $\left(x_{1}, y_{1}, z_{1}\right)$, it has a sequence $\left(x_{n}, y_{n}, z_{n}\right)$ of integral solutions, which in most cases corresponds to a compact set of points on the curve $F=0$. The proof of this is most easily obtained from elliptic functions; there ought to be a purely Diophantine proof, but the difficulty is that we have to estimate the "nearness" of a solution $\left(x^{\prime}, y^{\prime}, z^{\prime}\right)$ to a solution $(x, y, z)$, and $\left(x_{2}, y_{2}, z_{2}\right)$ in the sequence is not generally "near" to $\left(x_{1}, y_{1}, z_{1}\right)$ in the geometrical sense. Again, if we have a unicursal surface, such as that given by the parametric equations,

$$
x=\frac{q(\lambda \mu+1)}{\lambda+\mu}, \quad y=\frac{b(\lambda-\mu)}{\lambda+\mu}, \quad z=\frac{c(\lambda \mu-1)}{\lambda+\mu},
$$

whence $x^{2} / a^{2}+y^{2} / b^{2}-z^{2} / c^{2}=\mathrm{I}$, this suggests corresponding Diophantine theorems. Then, too, we have to consider solutions which, though not integral in the ordinary sense, are integral in certain algebraic fields; for instance, if $2 \rho=-\mathrm{I}+i \sqrt{ } 3$, then $\left(25^{\circ}-6 \rho, 1-9 \rho, 8+30 \rho\right)$ is a solution of $x^{3}+7 y^{3}-z^{3}=0$, which is integral in the field $(\rho)$, although it is not so in the field (I). In the latter field we have the solution $(I, I, 2)$; the reader is left to discover whether there are any other ordinary integral solutions, and if so, how many.

There are numerous exercises in the book which ought to stimulate the reader; some of them are practically suggestions for research. As a rule, it is unfair to expect a mathematical writer to give exact references to the sources of his examples; but in this case we wish Prof. Carmichael had been a little more definite, because in this subject even a short note on a very special problem may possibly contain the germ of an important discovery. As an instance of what we mean, Eisenstein's proof of the irreducibility of $\left(\mathrm{I}-x^{p}\right) /(\mathrm{I}-x)$, when $p$ is prime, is based on a theorem of his which must surely admit of some generalisation. To find whether any given polynomial is irreducible or not is practically such a laborious task (though theoretically possible) that special theorems like Eisenstein's are always welcome.

We hope that this book will have a wide circulation among mathematicians of all ages and capacities; it is rather a disgrace to the moderns that in this field they have added so little to the work of that great triumvirate, Diophantus, Fermat, and Euler. 